\title{
Setting Up Workflow of an Intraoperative MRI Unit: A Single-Centre Experience of First 53 Cases
}

\author{
Nitin Manohar ${ }^{1}$ Deviprasad Mohapatra ${ }^{1}$ Anandh Balasubramaniam² Keerthi Rao ${ }^{3}$ \\ Deepti Srinivas ${ }^{4}$ Dhritiman Chakrabarti ${ }^{5}$
}

${ }^{1}$ Department of Neuroanesthesiology, Yashoda Hospitals, Secunderabad, Telengana, India

${ }^{2}$ Department of Neurosurgery, Yashoda hospitals, Secunderabad, Telengana, India

${ }^{3}$ Department of Anaesthesiology, Rainbow Children's Hospital, Hyderabad

${ }^{4}$ Department of Neuroanaesthesia and Neurocritical Care, Apollo Hospitals, Bengaluru, Karnataka, India

${ }^{5}$ Department of Neuroanaesthesia and Neurocritical care, National Institute of Mental Health and Neuro Sciences, Bengaluru, Karnataka, India

\author{
Address for correspondence Deepti Srinivas, MD, Department \\ of Neuroanaesthesia and Neurocritical Care, Apollo Hospitals, \\ Bengaluru-560076, Karnataka, India \\ (e-mail: bsdeepti@gmail.com).
}

\begin{abstract}
Keywords

- intraoperative magnetic resonance imaging

- operating room magnetic resonance imaging

- image-guided neurosurgery

Background Setting up of a new "nearby" operation room type intraoperative magnetic resonance imaging (iMRI) unit poses a unique set of challenges to the operating team. We describe here an account of our experiences and a step by step protocol designed by us and followed to troubleshoot the issues encountered. The primary objective of the study was to observe our learning curve in the process of setting up of a new iMRI unit. The secondary objectives were to look at the number of residues detected, quality of imaging, and complications during iMRI.

Materials and Methods An observational study was conducted over a 3-month period involving cases requiring iMRI. Initially, a simulation was performed using healthy volunteers, which helped in developing a systematic protocol and drafting checklists to ensure a smooth workflow pattern. Data collection included details regarding hindrances encountered, how these were tackled, iMRI details (residual tumor, re-surgery), and complications, if any.

Results A total of 53 cases underwent iMRI in the study period. Among these, 51 were tumor cases that revealed residue (detected in 28 [54.9\%] cases), and re-surgery for further resection was performed in $21 / 28$ (75\%) cases. A very high level of surgeons' satisfaction regarding image quality of intraoperative scan was recorded (45/53 [84\%]). The number of personnel required for shifting and shifting times could be reduced with efficient utilization of the checklist.

Conclusion A methodical approach to tackle impediments while setting up a new unit such as iMRI facilitates its smooth functioning and ensures minimal interruptions and evades undue complications.
\end{abstract}

\section{Introduction}

Intraoperative magnetic resonance imaging (iMRI) technology is a landmark development in this era of image-guided

received

May 3, 2018

accepted after revision

August 28, 2018

published online

October 10, 2018

$10.1055 / \mathrm{s}-0038-1673503$ ISSN 2348-0548. neurosurgery. The important advantages of iMRI are that it provides real-time imaging, locates abnormalities in case of brain shift, distinguishes abnormal brain tissue from normal, and guides complete and precise resection of tumor,

C)2018 Indian Society of Neuroanaesthesiology and Critical Care

License terms 
thus minimizing residue and recurrence. ${ }^{1,2}$ Nevertheless, this technology involves a lot of challenges for the neuroanesthesia team involved with performing such cases. ${ }^{3}$ We share our experience of setting up a $3 \mathrm{~T}$, nearby operation room (OR) type iMRI unit and also present an audit of the cases we operated upon in the first 3 months including the challenges faced. The aim of our study was to identify impediments and tackle them optimally during the process of setting up and functioning of a new iMRI unit. The primary objective of the study was to observe our learning curve (duration of shifting time to and from the iMRI suite, and number of personnel required). The secondary objectives were to look at the number of residual tumors detected, uality of imaging, and complications during iMRI.

\section{Materials and Methods}

The iMRI setups can be of three types. First type comprises portable iMRI device which moves into the OR for imaging. In the second type, also called "nearby iMRI" suite, the MRI magnet is placed adjacent to the OR and patient is transported to the MRI suite for imaging. In the third type of iMRI set up, the MRI scanner is installed within the OR, and the MRI table can itself be used as the operating table.

Our setup is a nearby OR type, 3T-iMRI with the Siemens Skyra magnet located adjacent to the standard OR. This observational study of our first 53 cases during the first 3 months was performed to assess our learning curve of optimal resource utilization and conduct of cases using iMRI for the duration of October to December 2017. After installing the iMRI suite, mock drills were performed for 2 weeks to develop a protocol for shifting the patient to the iMRI, performing the scan, and shifting back to the operating room. Healthy volunteers were made to lie down on the operating table, and the maneuver of transporting the volunteer to the MRI suite and back was performed multiple times. The sequence of entry into the MRI suite was changed three times until the most comfortable sequence was finalized. All the personnel including scrub nurses and OR and MRI technicians who would be part of the team for iMRI were formally trained in didactic and practical session formats regarding the precautions and protocols to be followed. Warning signs and checklists were put on the entry door leading from OR to the MRI suite stating the MRI magnet would always be on and list of items prohibited inside the MRI suite at any time. Training was also conducted in categorizing all possible equipment, which could be present around and within the iMRI suite as MRI safe, unsafe, and conditional. A 5-Gauss line indicating strong magnetic field was marked inside the MRI suite. After the completion of training, actual surgical cases were started.

The surgeries were performed on a special operating table designed with an in-built MRI-compatible sliding board for moving the patient on to the MRI gurney intraoperatively. MRI coil was placed beneath the patient's head at the area of interest. Portable MRI compatible monitors were attached, and special MRI compatible carbon lead electrocardiogram (ECG) electrodes with braided wires were used. In the cases requiring evoked potential/electromyography monitoring, the subdermal needle/corkscrew electrodes used were "MR-conditional." Darcey et al tested the safety of subdermal needle and corkscrew electrodes in healthy volunteers and reported nonpainful heating and transient irritation in two and one site, respectively, of the 12 sites placed. ${ }^{4}$ They concluded that the MR-conditional electrodes can be used safely with iMRI. After induction of anesthesia, all skin to skin contact interfaces such as finger web spaces and perineum were padded with cotton pledgets to prevent moisture accumulation and heating during MRI, which can cause burns. The patients were then positioned for surgery. MRI compatible head pins and navigation system were used while positioning (-Fig. 1). After final positioning, a bore gauge was used to mimic the circumference of space available within the MRI magnet to confirm that the patient would slide smoothly in and out of the magnet, (-Fig. 1) especially in patients positioned prone and lateral. After this step, the surgeon was asked to proceed with the surgery.

When the surgeon felt the need for an iMRI scan, the MRI technician and radiologist were alerted at least 30 minutes prior to the expected time for beginning the scan. The MRI suite was cleaned and sterilized during this time. The patient and surgical site were packed and covered with sterile dressing without closing dura so as to allow residual tumor resection after iMRI (-Fig. 2a). Long intravenous (IV) line extensions (200 cm) and long breathing circuit extensions ( $1.5 \mathrm{~m} \times 2$ nos.) were connected to facilitate movement during MRI. MRI compatible infusion pump casing to carry infusion pumps during MRI was kept ready. Standard monitoring was continued with a portable TESLA MRI-compatible monitor for continuous monitoring of ECG, peripheral capillary oxygen saturation $\left(\mathrm{SpO}_{2}\right)$, end-tidal carbon dioxide $\left(\mathrm{EtCO}_{2}\right)$, and arterial blood pressure. A checklist for the patient and personnel going into the MRI suite was prepared during our training sessions to ensure patient, personnel, and equipment safety (-Fig. 3). The checklist was read and confirmed by the anesthesiologist every time before moving the patient to the MRI

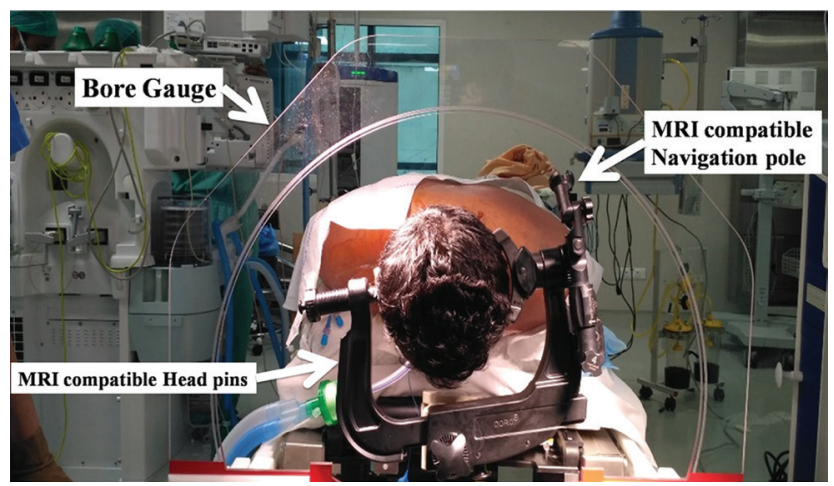

Fig. 1 MRI compatible bore gauge to mimic the size of the MRI gantry and MRI compatible head pins and navigation pole. MRI, magnetic resonance imaging 


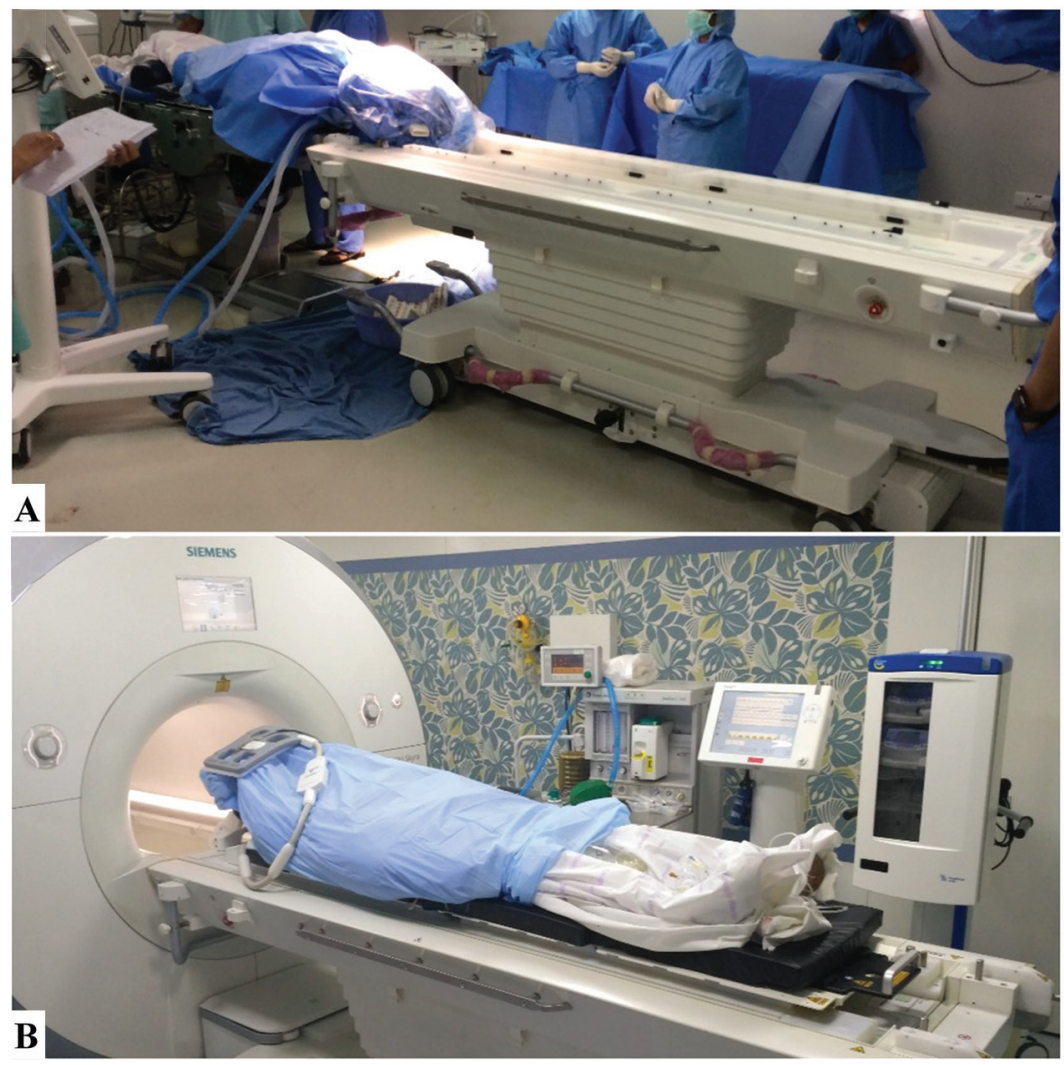

Fig. 2 (A) MRI gurney docked with OT table before the patient is packed in a sterile fashion and OT tabletop slid on to the MRI gurney. (B) Patient on the MRI gantry with upper coil placed on top of the area of interest for scan. MRI compatible anesthesia workstation, portable monitor, and infusion pump casing can be seen. MRI, magnetic resonance imaging; OT, operation theater.

suite. On completion of the checklist, MRI compatible gurney was brought in, docked with the operating table; and the patient was slid on to the gurney $(-$ Fig. 2a). The MRI compatible anesthesia workstation (GE Aestiva MRI; Datex Ohmeda Inc., Madison, Wisconsin, United States) in the MRI suite was checked and kept ready before moving the patient to the MRI suite ( $\mathbf{- F i g}$. $\mathbf{2 b}$ ). The breathing circuit was disconnected from the anesthesia machine in the operation theater and connected to the MRI compatible anesthesia workstation in the MRI suite. MRI compatible laryngoscope was checked and kept ready. Patient was taken into the MRI suite and during MRI scan, monitoring of vital parameters of the patient was continued, and a uniform depth of anesthesia was maintained ( - Fig. $2 b$ ). On completion of MRI, the MRI gurney with patient was brought back, re-docked to the operation table, and patient was moved on to the operation table. Any residual tumor detected on iMRI scan was removed or closure was performed after achievement of hemostasis.

Every time an iMRI was conducted, we recorded data regarding any mistakes, events, or complications while shifting the patient to the scan and back and during the scan, time required to move the patient into and out of the MRI suite, duration of the MRI scan, and number of personnel required to perform the iMRI scan. We also noted the number of patients in whom residue was detected and subsequently re-surgery for further resection was performed.

The data tabulation and calculation of descriptive statistics was conducted using Microsoft Excel 2010. Interval data were expressed as mean \pm standard deviation, and categorical data were expressed as frequencies and percentages.

\section{Results}

This observational study was conducted over 3 months from October 2017 to December 2017 involving the first 53 cases who underwent neurosurgery with iMRI at our institute. As this study included an intraoperative noninvasive diagnostic procedure and the objectives of interest pertained to conduct of the procedure itself, the institute's ethics committee waived off the need for a formal informed consent.

- Table 1 shows the demographic data of the patients along with the diagnosis and position of the patient. Of the 53 cases that underwent iMRI, 51 were for tumor resection, and the rest 2 cases were patients of Parkinson's disease for confirming position of deep brain stimulator (DBS) electrodes in the subthalamic nucleus. The most common surgeries for iMRI usage were transnasal trans-sphenoidal resection of pituitary tumors $(26 / 53 ; 49.03 \%)$ and cerebellopontine angle tumor resections $(12 / 53 ; 22.64 \%)$. Other surgeries in decreasing order of frequency were supratentorial tumor, posterior 


\section{CHECKLIST FOR INTRA-OP MRI:}

(To be Completed mandatorily prior to Wheeling-In; Please Tick on the boxes on checklist completion)

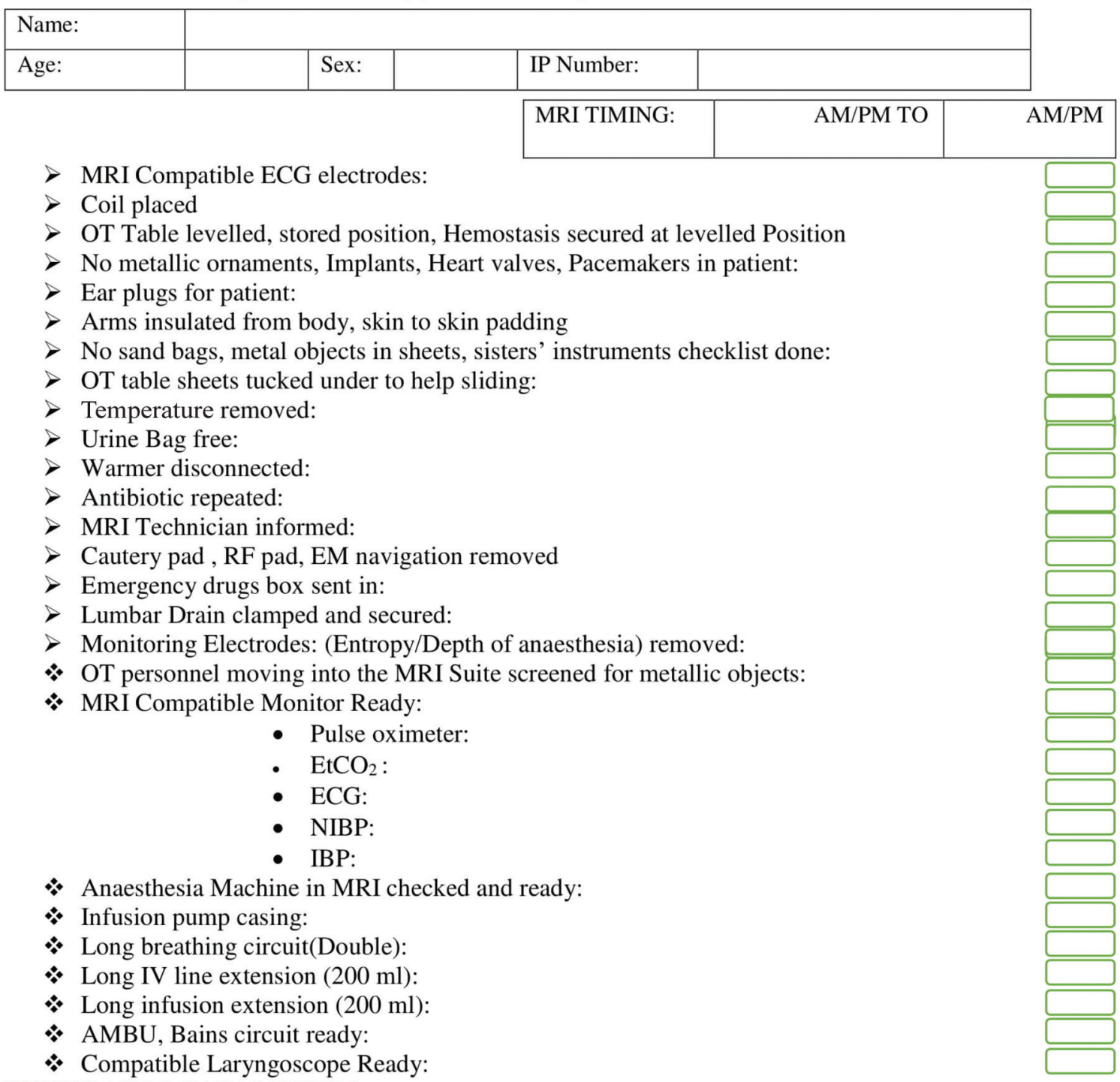

VENTILATOR PARAMETERS:

Ventilation Mode:

\begin{tabular}{|l|l|l|l|l|}
\hline TV & RR & $\mathrm{FiO}_{2}$ & PEEP & SEVOFLURANE \\
\hline & & & & \\
\hline
\end{tabular}

\section{INFUSIONS:}

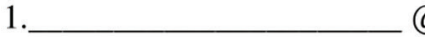

2.

a $\quad \mathrm{ml} / \mathrm{Hr}$

$3 . \mathrm{ml} / \mathrm{Hr}$

\section{While shifting:}

Urine Bag Free, Lines Free, Circuit Free

INFUSION PUMP WITH
PROTECTIVE CASING
MONITOR

Fig. 3 Checklist of our institute for patient, OT personnel and equipment safety to be performed before shifting for iMRI scan by the anesthesiologist. AMBU, artificial manual breathing unit; $\mathrm{ECG}$, electrocardiogram; $\mathrm{EM}$, electromagnetic; $\mathrm{EtCO}_{2}$, end-tidal carbon dioxide; $\mathrm{FiO}_{2}$, fraction of inspired oxygen; IBP, invasive blood pressure; iMRI, intraoperative magnetic resonance imaging; MRI, magnetic resonance imaging; NIBP, noninvasive blood pressure; OT, operation theater; PEEP, positive end-expiratory pressure; RF, radiofrequency; RR, respiratory rate; TV, tidal volume 
Table 1 Patient demographics, diagnosis, and intraoperative position of the patient

\begin{tabular}{|c|c|}
\hline Age (mean $\pm S D$ ) years & $50.26 \pm 15.32$ \\
\hline Sex (male:female) & $28: 25$ \\
\hline \multicolumn{2}{|l|}{ Diagnosis } \\
\hline Pituitary tumors & $26 / 53(49.03 \%)$ \\
\hline Cerebellopontine angle tumors & $12 / 53(22.64 \%)$ \\
\hline Posterior fossa tumors & $3 / 53(5.66 \%)$ \\
\hline Supra tentorial tumors & $8 / 53(15.09 \%)$ \\
\hline Arteriovenous malformations & $2 / 53(3.77 \%)$ \\
\hline $\begin{array}{l}\text { Parkinson's disease (for deep } \\
\text { brain stimulation) }\end{array}$ & $2 / 53(3.77 \%)$ \\
\hline \multicolumn{2}{|l|}{ Position } \\
\hline Supine & 38 \\
\hline Lateral & 12 \\
\hline Prone & 3 \\
\hline Awake craniotomies & 4 \\
\hline
\end{tabular}

Note: The total number of procedures conducted is 53 .

fossa tumors, and arteriovenous malformation resections. A special mention is its use during awake craniotomy for intracranial tumor resection.

Out of the 51 tumor cases, residue was detected in 28 (54.90\%) cases and no residue in 23 (45.09\%) cases. Of the cases where residual tumor was detected on iMRI, re-surgery for further resection to achieve maximal or total resection was performed in $21 / 28$ (75\%) cases, whereas in 7 cases the residual tumor was not chased as it was very close to a blood vessel or a critical eloquent structure; thus, subtotal excision was performed (-Table 2 ). The image quality of the scans was graded in a subjective manner on a 3-point scale based on the operating surgeon; in $45 / 53$ (84\%) cases, it was graded as excellent and provided vital information during performance of surgery (-Table 2). In eight cases, surgeons were not satisfied with the image quality of iMRI scan. Image quality was rated poor in two cases in prone position and average in six cases with intraoperative neurophysiological monitoring (somatosensory and motor evoked potentials) due to the corkscrew scalp electrodes causing artifacts.

We were able to cut down on the number of personnel required to shift patients in and out of iMRI after multiple iterations of the procedure. After initial cases, only four personnel (one anesthesia consultant, one anesthesia resident, one anesthesia technician, and one MRI technician) were required to shift the patients and perform the scan. Total duration of MRI scan time was reduced after initial cases as the number of requisite sequences were reduced as per need. The mean duration of time for shifting patients for the iMRI, mean scan duration, and time required for shifting back are tabulated in - Table 2 . The shifting times to and from the iMRI suite reduced after first few trials ( $\sim$ Fig. $4 \mathbf{A}$ and $\mathbf{B}$ ). We also analyzed and compared the shifting time and scan duration in terms of first 26 and next 27 cases ( - Table 2 ). Although both the shifting and scan duration were reduced in the last 27 cases, the shifting duration significantly reduced $(p=0.014)$. We also prepared a thorough checklist for ensuring patient, personnel, and equipment safety during patient transport as well as during the scan. This checklist was changed five times as and when issues were identified.

We also encountered complications during these cases. One patient had burns in prone position where the lower coil was touching the patient's nose, which was probably due to moisture collection. After this, we modified our technique in prone position and placed the lower coil just before moving into MRI scan to prevent blood and moisture collection. One patient had a swelling at the intravenous catheter site, after which we made it a protocol to make loops of IV line tubing to prevent cannula displacement due to tension on the IV line. Circuit got disconnected in one patient with a brief increase in $\mathrm{EtCO}_{2}$ while moving into the scan, and corrective measures were taken by adding a step of "tightening of circuit connections before shifting" to the shifting workflow, to prevent recurrence in further cases. We also noted logistical issues during each step of iMRI and planned for corrective measures so that that they would not recur in future cases. These were then incorporated while preparing the checklist.

\section{Discussion}

Though intraoperative MRI provides vital information to the neurosurgeons in planning and performing neurosurgeries with precision, it poses a lot of challenges for the anesthesia team. Safety of the patient, OT personnel, and equipment are core issues during such diagnostics. With the help of our training sessions and data collection, we were able to develop an institutional protocol for effective resource utilization in terms of time taken and personnel required. The time taken and the number of personnel reduced sequentially over the course of the first few scans, as the technique was improved upon iteratively.

Challenges faced by the neuroanesthesia team are centered around moving the patient intraoperatively on head pins, monitoring and maintaining hemodynamics during the shifting procedure, and maintaining adequate anesthetic depth and adequate ventilation during the movement of patient into the MRI suite and back. Issues about MRI compatibility of equipment. ${ }^{5,6}$ Training of staff involved, and other OT personnel should be conducted and coordinated by the anesthesiologist who should be given the role of team leader. ${ }^{7}$ Team work and inter-departmental communication is key to the success of any iMRI unit. ${ }^{8,9}$

Institutional protocols ${ }^{10-12}$ and checklists should be prepared ${ }^{13}$ and strictly adhered to for each case. ${ }^{14,15}$ Continuous data recording and auditing helped us reduce mistakes, personnel requirement, and also time taken to conduct the iMRI. ${ }^{16}$ Learning points from our experience in this regard to ensure smooth functioning of the setup have been summarized ( - Table 3 ). 
Table 2 Descriptive statistics of all collected data

\begin{tabular}{|c|c|}
\hline Time to start shifting till time to start scan (mean \pm SD) min & $7.26 \pm 6.50$ \\
\hline Duration of scan (mean \pm SD) min & $26.0 \pm 9.09$ \\
\hline Duration to shift patient back from MRI suite to OR (mean $\pm S D)$ min & $4.14 \pm 2.96$ \\
\hline Total duration including shifting in and out of MRI suite and scan time (mean \pm SD) min & $37.40 \pm 13.50$ \\
\hline Total duration required for shifting the patient in and out of MRI suite (mean \pm SD) min & $11.40 \pm 9.01$ \\
\hline Shifting duration: first 26 cases (mean \pm SD) min & $14.23 \pm 11.27$ \\
\hline Last 27 cases (mean \pm SD) min & $8.15 \pm 3.80$ \\
\hline p-Value & 0.014 \\
\hline Total duration-first 26 cases (mean \pm SD) min & $38.5 \pm 16.26$ \\
\hline last 27 cases (mean \pm SD) min & $35.29 \pm 9.77$ \\
\hline$p$-Value & 0.39 \\
\hline Total number of personnel required (median) & 4.00 \\
\hline \multicolumn{2}{|l|}{ Residue } \\
\hline Yes & $28 / 51(54.90 \%)$ \\
\hline No & $23 / 51(45.09 \%)$ \\
\hline Re-surgery for residue & $21 / 28(75 \%)$ \\
\hline Correct position of DBS electrode & $2 / 2$ \\
\hline \multicolumn{2}{|l|}{ Image quality } \\
\hline Excellent & 45 \\
\hline Average & 6 \\
\hline Poor & 2 \\
\hline \multicolumn{2}{|l|}{ Complications } \\
\hline Burns & 1 \\
\hline Circuit disconnection during shifting and rise in $\mathrm{EtCO}_{2}$ & 1 \\
\hline Hemodynamic disturbances & 3 \\
\hline Hypertension and tachycardia & 1 \\
\hline Hypotension & 2 \\
\hline Hematoma detected on iMRI & 1 \\
\hline IV line displaced with local swelling & 1 \\
\hline Small technical and corrective issues & 18 \\
\hline Issue during OT table docking & 5 \\
\hline During shifting into scan & 4 \\
\hline Docking into MRI & 1 \\
\hline During MRI scan & 4 \\
\hline Shifting back to OT and re-docking & 3 \\
\hline Charging of MRI compatible monitor: & 1 \\
\hline
\end{tabular}

Abbreviations: DBS, deep brain stimulator; $\mathrm{EtCO}_{2}$, end-tidal carbon dioxide; iMRI, intraoperative magnetic resonance imaging; MRI, magnetic resonance imaging; OR, operation room; OT, operation theater; SD, standard deviation.

\section{Conclusion}

While setting up any new interventional or investigative modality has its challenges, iMRI set up is a multidepartmental effort with significant issues pertaining to resource utilization and patient and personnel safety. Our experience shows that it takes multiple iterations of the procedure, coupled with initial training sessions and meticulous data collection and auditing to smoothen out the workflow. 


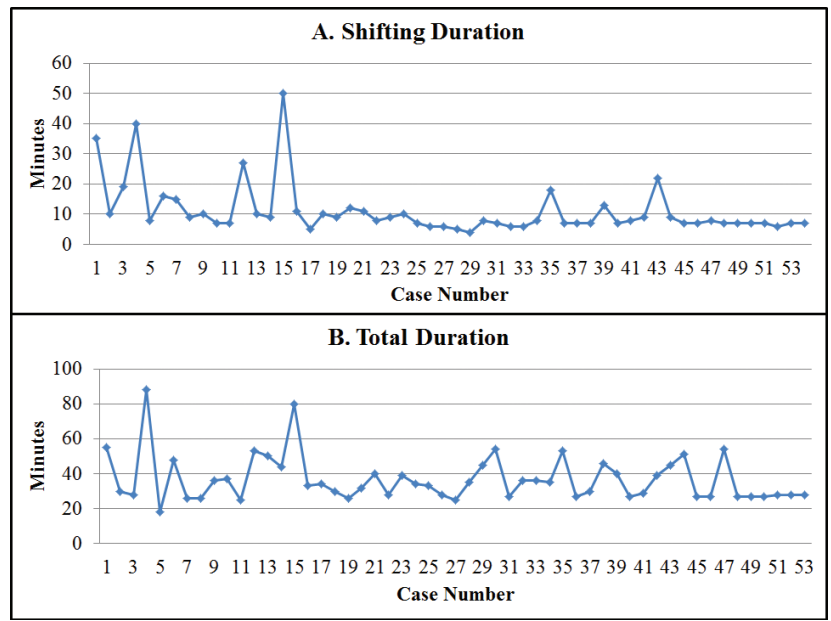

Fig. 4 Duration of shifting patients to and from iMRI suite $(\mathbf{A})$ and total duration of scan and shifting (B) of all patients $(n=53)$. iMRI, intraoperative magnetic resonance imaging.

Table 3 Learning points from our experience to ensure smooth functioning during setup of the new system

1. Categorize all equipment: MRI safe, unsafe, and conditional

2. Mock drills with healthy volunteers to develop initial protocol for shifting patients into iMRI and back to operating table.

3. Intensive training of all OT personnel-nursing staff, OR, and MRI technicians

4. Warning signs/checklists posted at strategic points

5. MRI compatible workstation/monitors/infusion pumpschecked prior to use

6. Burns prevention measures: cotton pledgets placement at skin to skin contact interfaces

7. Long intravenous extensions and breathing circuit with firm attachment and adequate slack to prevent disconnections

8. Strict adherence to detailed checklist customized as per institute practices and resources

Abbreviations: iMRI, intraoperative magnetic resonance imaging; MRI, magnetic resonance imaging; OR, operation room; OT, operation theater.

\section{Funding}

None.

\section{Conflict of Interest}

None.

\section{References}

1 Black PM, Moriarty T, Alexander E, et al. Development and implementation of intraoperative magnetic resonance imaging and its neurosurgical applications. Neurosurgery 1997;41(4):831-842, discussion 842-845

2 Black PM, Alexander E, Martin C, et al. Craniotomy for tumor treatment in an intraoperative magnetic resonance imaging unit. Neurosurgery 1999;45(3):423-431, discussion 431-433

3 Berkenstadt H, Perel A, Ram Z, Feldman Z, Nahtomi-Shick O, Hadani M. Anesthesia for magnetic resonance guided neurosurgery: initial experience with a new open magnetic resonance imaging system. J Neurosurg Anesthesiol 2001;13(2):158-162

4 Darcey TM, Kobylarz EJ, Pearl MA, et al. Safe use of subdermal needles for intraoperative monitoring with MRI. Neurosurg Focus 2016;40(3):E19

5 Archer DP, Manninen PH, Mctaggart-cowan RA. Anesthetic considerations for neurosurgery using intraoperative magnetic resonance imaging. Tech Neurosurg 2002;7(4):308-312

6 Schmitz B, Nimsky C, Wendel G, et al. Anesthesia during high-field intraoperative magnetic resonance imaging experience with 80 consecutive cases. J Neurosurg Anesthesiol 2003;15(3):255-262

7 Tan TK, Goh J. The anaesthetist's role in the setting up of an intraoperative MR imaging facility. Singapore Med J 2009;50(1):4-10

8 Netuka D, Masopust V, Belšán T, Kramář F, Beneš V. One year experience with $3.0 \mathrm{~T}$ intraoperative MRI in pituitary surgery. Acta Neurochir Suppl (Wien) 2011;109:157-159

9 Chen X, Xu BN, Meng X, Zhang J, Yu X, Zhou D. Dual-room 1.5-T intraoperative magnetic resonance imaging suite with a movable magnet: implementation and preliminary experience. Neurosurg Rev 2012;35(1):95-109, discussion 109-110

10 Ginat DT, Swearingen B, Curry W, Cahill D, Madsen J, Schaefer PW. 3 Tesla intraoperative MRI for brain tumor surgery. J Magn Reson Imaging 2014;39(6):1357-1365

11 Zheng X, Xu X, Zhang H, et al. A preliminary experience with use of intraoperative magnetic resonance imaging in thalamic glioma surgery: a case series of 38 patients. World Neurosurg 2016;89:434-441

12 Berkow LC. Anesthetic management and human factors in the intraoperative MRI environment. Curr Opin Anaesthesiol 2016;29(5):563-567

13 Rahmathulla G, Recinos PF, Traul DE, et al. Surgical briefings, checklists, and the creation of an environment of safety in the neurosurgical intraoperative magnetic resonance imaging suite. Neurosurg Focus 2012;33(5):E12

14 Johnston T, Moser R, Moeller K, Moriarty TM. Intraoperative MRI: safety. Neurosurg Clin N Am 2009;20(2):147-153

15 Bergese SD, Puente EG. Anesthesia in the intraoperative MRI environment. Neurosurg Clin N Am 2009;20(2):155-162

16 Marongiu A, D’Andrea G, Raco A. 1.5-T field Intraoperative Magnetic Resonance Imaging Improves Extent of Resection and Survival in glioblastoma removal. World Neurosurg 2017;98:578-586 